\title{
Die Größe macht's
}

\author{
WISSENSCHAFT ERKLÄRT: EFFEKTGRÖSSEN Oft fragen sich Therapeuten, welche \\ Behandlungsmethode für welchen Patienten am effektivsten ist. Beim Lesen von \\ wissenschaftlichen Studien hilft dabei ein Blick auf die Effektgröße. Was eine Effekt- \\ größe beschreibt, lesen Sie in diesem Artikel.
}

E

in Therapeut sollte immer versuchen, die Behandlungsmethode anzuwenden, die für Patienten am effektivsten ist. In der Wissenschaft gibt es mathematische Möglichkeiten, einen Therapieeffekt zu berechnen. Doch wie erkennt ein Physiotherapeut, ob in einer Studie von einem eindeutigen Therapieeffekt die Rede ist?

Verschiedene Tests vergleichen > Ein Physiotherapeut behandelt einen Patienten nach Schlaganfall bezüglich seiner Einschränkungen im Arm- und Schulterbereich. Dies tut er nach einem altbewährten Therapiekonzept. Dass diese Therapiemethode effektiv ist, hat er in einer Studie gelesen. Forscher haben mithilfe des Fugl-Meyer-Tests herausgefunden, dass besagtes Therapiekonzept einen positiven Effekt auf die Armfunktionen der Patienten hat. Als der Physiotherapeut von einer Studie zu einer neuen Behandlungsmethode hört, die auch klinisch bedeutsam sein soll, wird er neugierig. Die untersuchte Methode verspricht nicht nur Schmerzreduktion für Patienten nach einem Schlaganfall, sondern auch eine bessere Armfunktion, erhöhte Alltagskompetenz und mehr Lebensqualität. Sie beschreibt Ergebnisse des Box \& Block-Tests und des Amount of Use Tests (AOUT), die zuverlässig und valide die Schulter- und Armfunktionen messen.

Wie kann der Physiotherapeut jetzt die Ergebnisse der zwei Studien mit unterschiedlichen Tests vergleichen? Woher weiß er, ob die neue Methode einen besseren Effekt hat? Der Psychologe Jacob Cohen hat dazu bereits 1969 eine nützliche Berechnung von sogenannten Effektgrößen (auch: Cohens d, ES oder effect size) entwickelt:

$\mathbf{d}=\frac{\left(\text { Mittelwert }_{1}-\text { Mittelwert }_{2}\right)}{\text { Standardabweichung }}$

\section{Auskunft über den Effekt > Diese Formel} geben Forscher in wissenschaftlichen Studien oft für die verschiedenen Tests an. Interessant für den Leser dieser Studien ist weniger die Formel als das Ergebnis der Berechnung. Es ist ein standardisiertes metrisches Maß, das man sowohl für Studien als auch für therapeutische Befundungen verwenden kann. Das Ergebnis gibt Auskunft über die Größe des Effektes einer Therapiemethode und ist immer größer 0 . Es kann auch deutlich größer 1 sein. 0,2 betrachtet Cohen als einen sehr kleinen Therapieeffekt, 0,5 beschreibt einen moderaten oder mittleren Therapieeffekt, und bei 0,8 ist der Therapieeffekt groß. Je höher der Wert, desto effektiver ist die Therapie.

Der Therapeut aus unserem Beispiel schaut sich die d-Werte noch einmal genauer an. Das neue Konzept hat einen d-Wert von 0,7, die alte Studie kam nur auf $d=0,56$. Da die neue Studie einen höheren Therapieeffekt verspricht, fühlt sich der Physiotherapeut ermutigt, besagtes Konzept an seinem Patienten zu testen.

Pearson als Alternative > Manche Studien geben keine Auskunft über Cohens d, sondern verwenden das sogenannte ein Pearsons-Zusammenhangsmaß. Dabei handelt es sich um eine Berechnung zum Zusammenhang, die ähnliche Auskunft gibt. Pearson verwendet anstelle des $d$ ein $r$, setzt aber die gleichen Maßstäbe: $r=0,2$ spiegelt einen kleinen Zusammenhangseffekt wider, 0,5 ist ein mittlerer Effekt, und bei 0,8 spricht er von einem großen Effekt. Pearsons $r$ ist immer zwischen -1 und 1 . Weitere Alternativen sind sogenannte standardisierte Mittelwertsdifferenzen.

Effektgröße in der Studienplanung $>$ In der Forschung spielt die Effektgröße noch eine andere Rolle: Bei der Planung einer Stu-

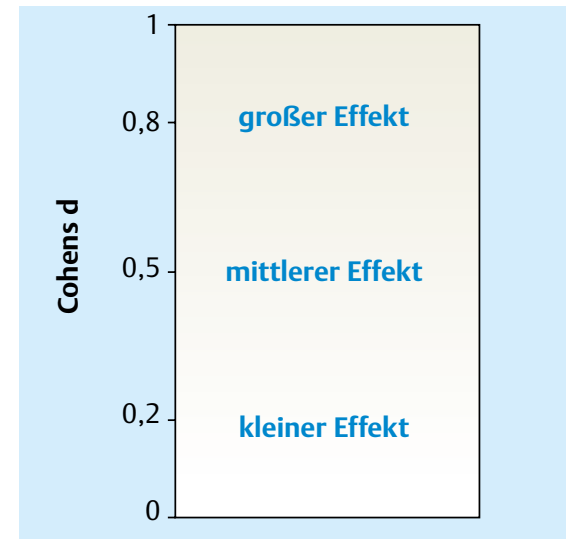

Je höher ein Wert einer Effektgröße ist, desto größer ist der Therapieeffekt für den Patienten.

die berechnet der Forscher unter anderem anhand der Effektgröße, wie hoch die Teilnehmerzahl seiner Studie sein muss, damit die Studie aussagekräftig sein kann. Dies geschieht mit speziellen Formeln und Programmen. Möchte man einen großen Therapieeffekt darstellen, benötigt man oftmals nur wenige Patienten. Mit der Berücksichtigung der Effektgröße bei der Studienplanung verhindert man unethische Untersuchungen.

Vergleicht man als Therapeut Studien miteinander, ist es empfehlenswert, sich unter anderem die Effektgrößen anzusehen, denn je näher Cohens d oder Pearsons $r$ an die Zahl 1 herankommen, desto größer ist der Effekt der Therapie.

Jan Mehrholz

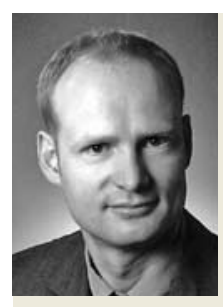

Prof. Dr. Jan Mehrholz erhielt dieses Jahr für seine Studie „Gehfähigkeit, Alltagskompetenz und Lebensqualität von Patienten nach Schlaganfall“ den Förderpreis des Neurologischen Rehabilitationszentrums Jugendwerk Gailingen e.V. 